\title{
Dianzani autoimmune lymphoproliferative disease
}

INSERM

\section{Source}

INSERM. (1999). Orphanet: an online rare disease and orphan drug data base. Dianzani autoimmune lymphoproliferative disease. ORPHA:275523

Dianzani autoimmune lymphoproliferative disease (DALD) is a very rare disorder characterized by autoimmunity, lymphadenopathy and/or splenomegaly. 